\title{
Coexistence of episomal and integrated HPV16 DNA in squamous cell carcinoma of the cervix
}

\author{
E Kristiansen, A Jenkins, R Holm
}

\begin{abstract}
Aims-To investigate the integration of human papillomavirus (HPV)16 in 13 HPV16 positive cervical squamous carcinomas.

Methods-Samples were investigated by Southern blot analysis of the Pst I digestion pattern, two-dimensional gelelectrophoresis, and in situ hybridisation.

Results-Integration of HPV16 was found in all cases. In 12 biopsy specimens episomal HPV16 DNA and integrated HPV16 DNA were seen. The episomal DNA occurred as dimers and multimers. In situ hybridisation showed that both integrated and episomal HPV16 DNA were present in the same cell in most tumour cell nuclei.
\end{abstract}

Conclusions-An intact episomal E2 gene is present in most cases of these cervical cancers, and could therefore replace the regulatory function of an integrated defective $\mathrm{E} 2$ gene.

(F Clin Pathol 1994;47:253-256)

Recent epidemiological studies have shown that most human cervical cancers harbour the "high risk" human papillomavirus (HPV) types HPV16, 18, 31, and 33. ${ }^{1} \mathrm{HPV} 16$ and 18 can immortalise primary human keratinocytes in culture. ${ }^{2-5}$ There is every reason to believe, therefore, that HPV is an important aetiological factor in cervical carcinogenesis.

HPV16 is the most common HPV type seen in cervical carcinomas and is often found to be integrated. As benign lesions mainly contain episomal HPV 16 DNA, ${ }^{67}$ integration of HPV into the human chromosome may be an important step in cervical carcinogenesis. High risk HPVs encode two oncogenic proteins E6 and E7; these are expressed in HPV positive cancers and in derived cell lines. 8 $^{-10}$ Expression of these proteins is regulated by one of the viral gene products from open reading frame (ORF) E2."11 Integration of HPV into the human chromosome disrupts the expression of the E1-E2 genes, ${ }^{12-15}$ thereby anulling E6 and E7 regulation. This could be an important step in the progression of HPV lesions to cancer.

There are three methods for the detection of integrated HPV DNA: restriction analysis of Southern blots; two-dimensional gelelectrophoresis; and in situ hybridisation. Restriction analysis relies on the fact that integration will alter the characteristic size of one of the viral restriction fragments, creating two new junction fragments. This method is most effective when a battery of restriction enzymes is used, as junction fragments are not always revealed in a single restriction digest. Twodimensional electrophoresis relies on the fact that linear and circular DNA have different relative mobilities in agarose gels of different concentrations. DNA is cut with a restriction enzyme that has no target sites in the virus genome. This leaves episomal viral DNA in intact circles, while integrated virus is found in linear fragments produced by cutting of the flanking host sequences. In the second dimension of electrophoresis circular and linear DNA separate into two tracks, allowing bands to be assigned to linear and circular DNA. Cooper et al ${ }^{16}$ have recently described a technique of detecting HPV integration using in situ hybridisation. These authors observed that in situ hybridisation signals may be spread over the entire nucleus (diffuse), concentrated in small spots (punctuate), or in a mixture of the two. They found that diffuse signals correspond to episomal DNA, while punctuate signals correspond to integrated DNA.

Integration studies of HPV in cervical carcinoma have given conflicting results. Several studies have shown that HPV is mainly integrated in cervical cancers, ${ }^{2717}$ cell lines derived from cervical cancers, ${ }^{8918}$ and in immortalised human keratinozytes. ${ }^{19} 20$ In contrast, Matsukura et al failed to detect integrated DNA in up to $70 \%$ of their cancer biopsy specimens. ${ }^{21}$ These results raise the question of whether integration is strictly necessary for malignancy to develop.

\section{Methods}

Biopsy specimens were snap frozen in liquid nitrogen immediately after collection. Each biopsy specimen was divided; one half was sent for histological examination and the other half was used for DNA analysis. ${ }^{22}$

The physical state of HPV was determined by two dimensional gelelectrophoresis. ${ }^{23}$ Biopsy DNA from cervical squamous carcinomas infected with HPV16 were digested with Hind III (an HPV non-cutter). Agarose gels of $0.5 \%$ and $1 \%$ were used for the first and second dimensions, respectively.

\section{DNA HYBRIDISATION}

DNA was transferred to the filter (Hybond N, Amersham) by a vacuum blotter (LKB), and prehybridised for two hours at $68^{\circ} \mathrm{C}$ in a hybridisation buffer containing $5 \times$ SSC 
( $0.75 \mathrm{M}$ sodium chloride, $0.075 \mathrm{M}$ sodium citrate), $5 \times$ SPEP ( $5 \%$ sodium dodecyl sulphate, $1 \%$ pyrophosphate, $0.05 \mathrm{M}$ EDTA, $0.010 \mathrm{M}$ sodium phosphate buffer, $\mathrm{pH} 6.8$ ), $50 \times$ Denhardt's solution, $30 \%$ Dextran solution; and $10 \mathrm{mg} / \mathrm{ml}$ salmon sperm. The probe was prepared by gel purification of HPV16 and labelled with ${ }^{32} \mathrm{P}$ using a multiprime DNA labelling system (Amersham). Hybridisation was continued for 12 to 16 hours in the same buffer and temperature as for prehybridisation. The filters were washed at $48^{\circ} \mathrm{C}$, twice in $2 \times$ SSC and $0.05 \%$ SDS, and then twice in $0.1 \times$ SSC and $0 \cdot 1 \%$ SDS.

\section{IN SITU HYBRIDISATION}

In situ hybridisation was carried out with biotinylated probes against HPV16 $6^{24}$ and an alkaline phosphatase anti-alkaline phosphatase (APAAP) detection system, as described by Holm et al. ${ }^{25}$ Briefly, sections from formalin fixed, paraffin-wax embedded material were treated sequentially with proteinase $\mathrm{K}(250 \mu \mathrm{g} / \mathrm{ml}), 0 \cdot 2 \%$ glycine, and $4 \%$ paraformaldehyde. After probe and cellular DNA were denaturated together by heating at $95^{\circ} \mathrm{C}$ for 10 minutes, the slides were hybridised for $18-22$ hours at $37^{\circ} \mathrm{C}$. Tissue sections were given high stringency washes before hybridised DNA was detected using sequential incubation with mouse antibiotin, rabbit anti-mouse IgG, and alkaline phosphatase-mouse anti-alkaline phosphatase. The alkaline phosphatase reactions were developed using nitroblue tetrazolium and 5bromo-4-chloro-3-indolyl-phosphate as chromogenes.

\section{DNA MOLECULAR MARKERS}

IDNA digested with Hind III and high molecular weight DNA markers (Bethesda Research Laboratories) were used as linear molecular weight markers. The supercoiled ladder marker (Bethesda Research Laboratories) and the bacteriophage P2 were used as circular DNA molecular markers.

\section{Results}

Seven of 13 cervical squamous cell carcinomas positive for HPV16 DNA by Southern blotting showed off sized bands consistent with integration when digested with Pst $I$ and probed with HPV16 DNA (data not shown). The other six samples showed Pst I fragments corresponding to circular HPV16 DNA.

To achieve a better assessment of the physical state of HPV in this biopsy collection, two-dimensional gelelectrophoresis was performed. Integrated HPV DNA is detected as a hybridisation signal on the linear track. Signals from the circular HPV DNA, however, appear above the linear track, due to a slower mobility in the second dimension. Typical results are shown in fig 1 .

Integrated HPV16 DNA was detected in all 13 cases; 12 samples also contained episomal DNA. One sample showed only integrated DNA. One out of 13 samples gave two hybridisation signals on the linear track (data not shown), indicating HPV16 integration at two different locations in the human chromosome. In one case, in addition to a linear fragment of 16 kilobases and an episome of 8 kilobases, a signal of extremely high molecular

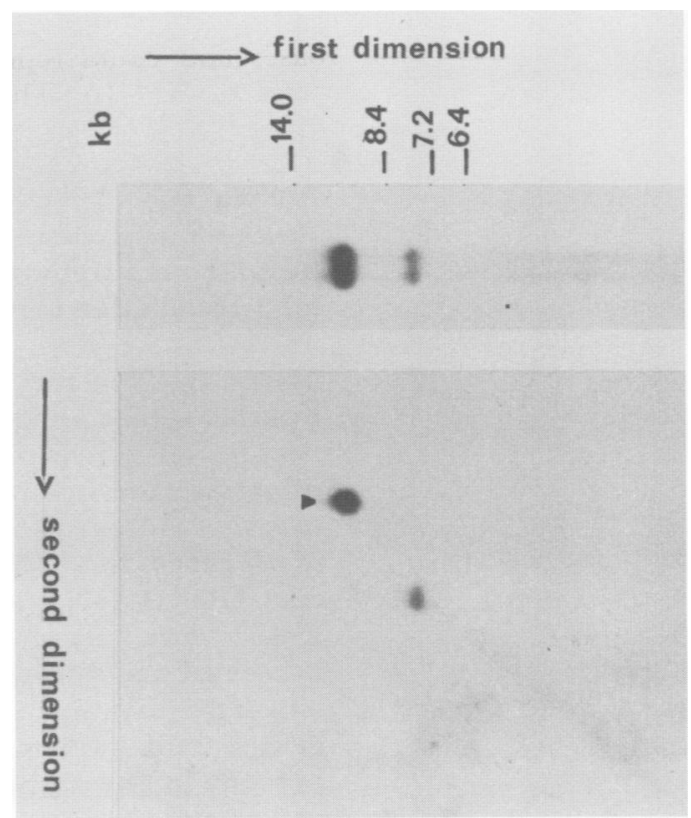

Figure 1 (A) Analysis of HPV16 DNA by twodimensional gelelectrophoresis. $20 \mu \mathrm{g}$ of total cell DNA were digested with Hind III and electrophorised twice at 30 volts for 24 hours each on $0.5 \%$ and $1 \%$ agarose gels respectively. Hybridisation was performed with a ${ }^{32} P$ labelled HPV16 probe. In the first dimension two bands of 8 and 12 kilobases can be detected. These were shown to belong to two groups by two-dimensional electrophoresis. In the second dimension the 8 kilobase fragment can be seen on the linear track. The upper fragment is seen on the circular track (arrowhead) corresponding to 16 kilobases on the supercoiled molecular weight standard (not shown). This sample contains episomal and integrated HPV16

DNA.

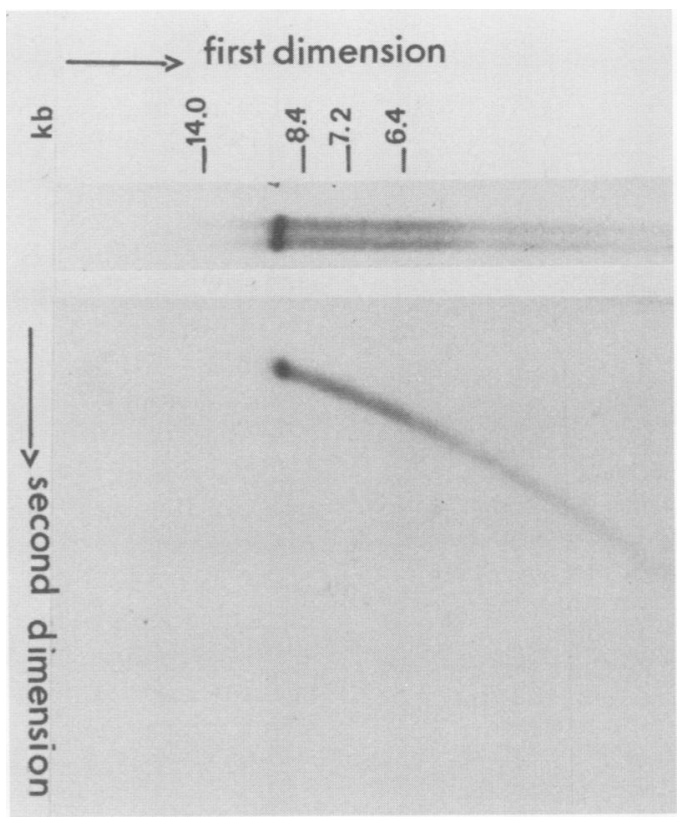

Figure 1 (B) One band of 9 kilobases can be observed in the first dimension. In the second dimension this band can be seen on the linear track, showing that this biopsy specimen contained integrated HPV16 DNA only. 
weight was observed. At such high molecular weights, the circular and linear tracks merge, so the physical state of the virus DNA could not be determined.

In 12 of 13 cases HPV 16 containing linear fragments ranged from 7 to 16 kilobases, indicating a maximum of two integrated copies. In one sample the size was more than 48 kilobases and could represent integrated multimers of HPV16. The episomal HPV DNA appeared in dimers and multimers; we did not detect any monomers. One sample harboured both multimeric and dimeric episomal forms. The sizes of the linear and the episomal forms were compared in each sample. In each case the linear fragment differed in size compared with the episomal form, indicating that linear DNA was not simply a breakdown product of circular DNA.

In situ hybridisation analysis of HPV16 DNA showed that in one of the 13 cases tested a punctuate signal was detected (fig $2 \mathrm{~A}$ ), whereas in 12 cases both a punctuate and a diffuse signal were identified (fig 2B). In these cases coexistence of punctuate and diffuse signals was found in most tumour cell nuclei. The 12 cases containing both punctuate and diffuse signals showed an integrated and episomal pattern using two-dimensional gelectrophoresis, whereas the only case with a punctuate signal gave an exclusively integrated pattern (fig 1B).

\section{Discussion}

In this study we have shown that 13 of 13 cases of cervical squamous carcinomas
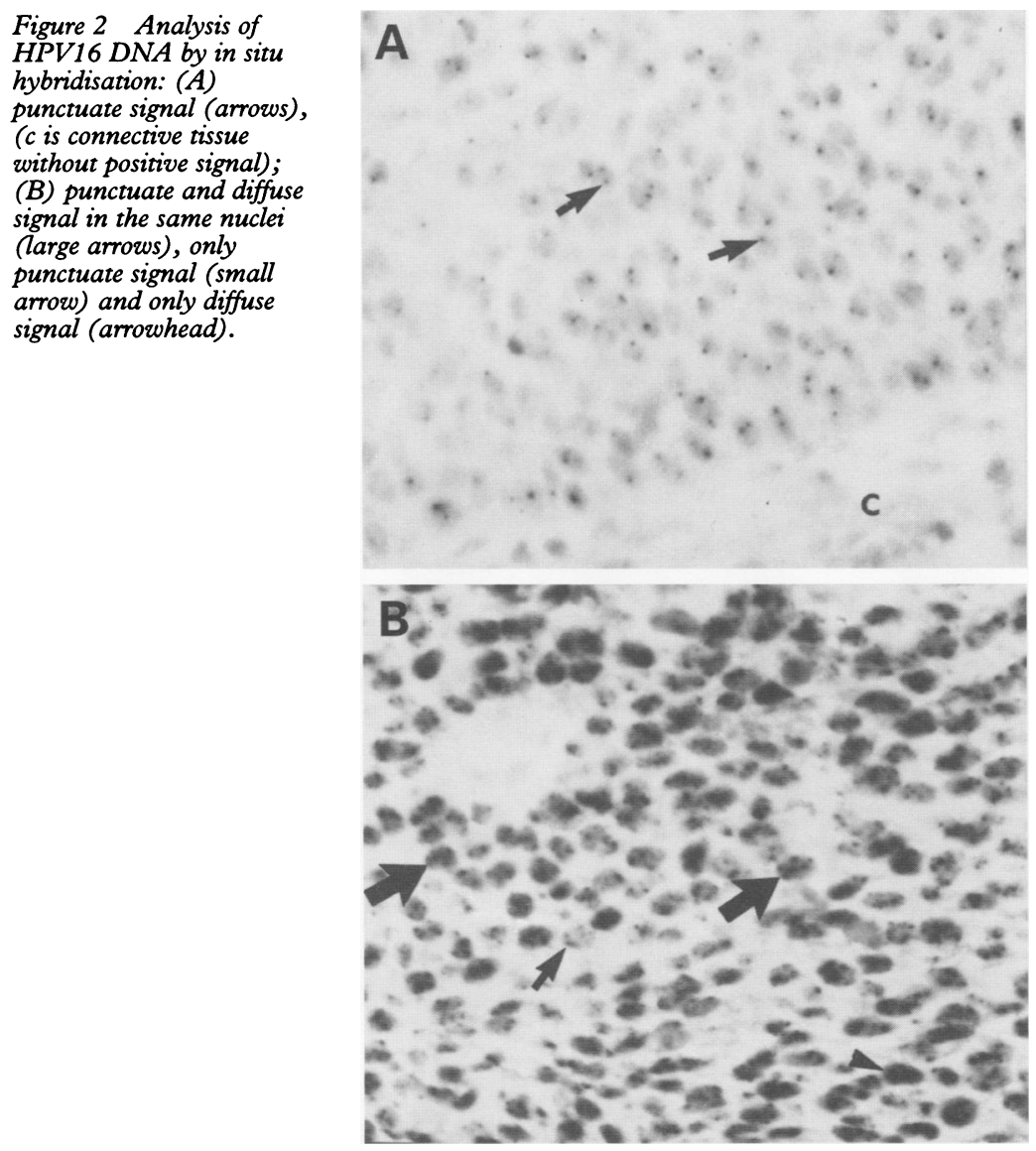

infected with HPV16 contained integrated HPV16 DNA; 12 of these samples also contained episomal forms. Episomal forms were present exclusively in dimeric and multimeric forms; monomeric episomes were not found. In 12 out of 13 cases the episomal form appeared together with the integrated form. In all cases the linear form had a different size from the episomal form, confirming that the linear forms observed were not randomly linearised episomal HPV. DNA damaged through DNA purification.

In situ hybridisation results confirmed the results shown by two-dimensional gelelectrophoresis. In addition, valuable data on the coexistence of episomal and integrated HPV16 DNA within the same cell nuclei were provided. Our results agree with a previous report that a punctuate signal represents integrated HPV DNA and a diffuse signal represents episomal HPV DNA. ${ }^{16}$

Studies on HPV integration have shown a wide variation in observed integration frequencies. Various combinations of restriction analysis and two-dimensional gelelectrophoresis have been used. In our results integration was detectable by Southern blotting in $54 \%$ of the samples, and in $100 \%$ by two-dimensional gelelectrophoresis or in situ hybridisation. This suggests that junction fragments are frequently masked behind the episomal Pst $I$ band pattern seen in Southern blotting and thereby interpreted only as episomal HPV DNA.

Our results also agree with other studies ${ }^{717}$ which found HPV16 integrated into most cervical cancers with episomal DNA. Matsukura et $a l^{21}$ and Fuchs et $a l^{22}$ found only episomal HPV 16 in $70 \%$ and $36 \%$ of cervical cancers, respectively. Our results are clearly at variance with these latter studies.

Coexistence of integrated and episomal HPV16 DNA in the same cancer cell may have some consequences for theories that seek to explain the high prevalence of viral integration in cervical cancers. It has been suggested that integration of HPV16 contributes to the maintenance of the transformed state by inactivating the viral E2 gene, thereby releasing the E6 and E7 oncogenes from control. This theory suggests that integrated and episomal HPV16 DNA would not coexist in the same cell, as an episomal form would then be able to complement the E2 defect of the integrated HPV16, thereby restoring control of the E6 and E7 genes. This hypothesis is therefore difficult to reconcile with our finding that coexistence of integrated and episomal HPV16 is normal in squamous cervical cancers. However, we did not investigate the pattern of RNA expression in our samples, so the possibility exists that the integrated HPV copies are transcribed and the episomal HPV copies are not transcribed as a consequence of, for instance, greater stability or translatability of RNA attached to flanking chromosomal sequences. In such a case unbalanced expression of E2 could persist even in the presence of intact episomes. It is possible, therefore, that HPV integration may have some other 
role in cervical carcinogenesis, such as activation of neighbouring cellular oncogenes, integrative inactivation of cellular tumour suppressor genes, or facilitating viral replication.

We thank Drs BH Lindqvist, C Trope, and BE Kristiansen for support. We are also grateful to Drs $\mathrm{H}$ zur Hausen and Ethelsupport. We are also grateful to Drs H zur Hausen and Ethel-
Michele de Villiers for samples of HPV16 DNA. This work was supported by a grant from the Medical Faculty, University of Oslo.

1 Reeves WC, Rawis WE, Brinton LA. Epidemiology of genital human papillomaviru

2 Durst M, Dzarlieva-Petrusevska RT, Boukamp P, Fusenig NE, Gissmann L. Molecular and cytogenetic analysis of immortalized human primary keratinocytes obtained immortalized human primary keratinocytes obtained after transfection with human

3 Pirisi L, Yasumoto S, Fellerey M, Doniger JK, DiPaolo JA. Transformation of human fibroblasts and keratinocytes with human papillomavirus type 16 DNA. $\mathcal{f}$ Virol 1987;61:1061-6.

4 Kaur P, McDougal JK. Characterization of primary human keratinocytes transformed by human papillomavirus type 18. F Virol 1988;62:1917-24.

5 Schlegel R, Phelps WC, Zhang Y-L, Barbosa M. Quantitative keratinocyte assay detects two biological activities of human papillomavirus DNA and identifies viral types associated with cervical carcinoma. Embo $\mathcal{f}$ viral types asso

6 Durst M, Kleinheinz A, Hotz M, Gissmann L. The physical state of human papillomavirus type 16 DNA in benign and malignant cervical tumours. $\mathcal{f}$ Gen Virol 1985;66:1515-22.

7 Cullen AP, Reid R, Campion M, Lorincz T. Analysis of the physical state of different human papillomavirus DNAs in intraepithelial and invasive cervical neoplasm. f Virol 1991;65:606-12.

8 Smotkin D, Wettstein FO. Transcription of human papillomavirus type 16 early genes in a cervical cancer and a cancer derived cell line and identification of the E7 and a cancer derived cell line and identification of

9 Yee C, Krishnan-Hewlett I, Baker CC, Schlegel R, Yee C, Krishnan-Hewlett I, Baker CC, Schlegel R,
Howley PM. Presence and expression of human papilloHowley PM. Presence and expression of human papillomavirus sequences in human cer
lines. $A m$ f Pathol 1985;119:361-6.

10 Schwartz E, Freese UK, Gissmann L, et al. Structure and transcription of human papillomavirus sequences in cervical carcinoma cells. Nature 1985;314:111-14.

11 Cripe TP, Haugen TH, Turk JP, et al. Transcriptional regulation of the human papillomavirus-16 E6-7 promoter by a keratinocyte-dependent enhancer, and by viral E2 trans-activator and repressor gene products: Implications fo 3745-53.

12 Schwartz E, Schneider-Gadicke A, Roggenbuch B, Mayer W, Gissmann L, zur Hausen H. Expression of human papillomavirus DNA in cervical carcinoma cell lines. papillomavirus DNA in cervical

13 Awady M, Kaplan J, O'Brien S, Burk R. Molecular analysis of integrated papillomavirus 16 sequences in the cervical of integrated papillomavirus 16 sequences in the

14 Choo KB, Pan CC, Han S. Integration of human papillomavirus type 16 into cellular DNA of cervical carcinoma: preferential deletion of the $\mathrm{E} 2$ gene and invariable retention of the long control region and the E6/E7 open reading frames. Virology 1987;161:259-61.

15 Choo K, Lee C, Pan S, et al. Sequence duplication and internal deletion in the integrated human papillomavirus type 16 genome cloned

16 Cooper $\mathrm{K}$, Herrington CS, Stickland JE, Evans MF McGee JO'D. Episomal and integrated human papilloMcGee JO'D. Episomal and integrated human papillomavirus in cervical neoplasia shown by non-1s

17 Fukushima M, Yamakawa Y, Shimano S, Hashimoto M Sawada Y, Fujinaga K. The physical state of human papillomavirus 16 DNA in cervical carcinoma and cervical intraepithelial neoplasia. Cancer 1990;66:2155-61.

18 Pater M, Pater A. Human papillomavirus types 16 and 18 sequences in carcinoma cell lines of the cervix. Virology 1985;155:313-18.

19 McDougall JK. Human papillomavirus immortalising and transformation. Papillomavirus Reports 1990;1:1-4.

20 Popescu NC, Dipaolo JA. Integration of human papillomavirus 16 DNA and genomic rearrangements in mavirus 16 DNA and genomic rearrangements in 1990;50:1316-23.

21 Matsukura T, Koi S, Sugase $M$. Both episomal and integrated forms of human papillomavirus type 16 are involved in invasive cervical cancers. Virology 1989; 172:63-72.

22 Fuchs P, Girardi F, Pfister H. Human papillomavirus 16 DNA in cervical cancers and in lymph nodes of cervical cancer patients: a diagnostic marker for early metastases? Int 7 Cancer 1989;43:41-4.

23 Kristiansen E, Jenkins A, Christensen G, et al. Human papillomavirus infection in Norwegian women with cervical cancers. APMIS (in press)

24 Wettstein FO, Stevens JG. Variable-sized free episomes of Shope papillomavirus DNA are present in all non-virusproducing neoplasms and integrated episomes are detected in some. Proc Natl Acad Sci USA 1982;79: detected

25 Holm R, Karlsen K, Nesland JM. In situ hybridization with non-isotopic probes using different detection systems. Mod Pathol 1992;5:315-20. 